\title{
Improvement in Psoriasis With Rosiglitazone in a Diabetic and a Nondiabetic Patient
}

\author{
Harrihar A. Pershadsingh, MD, PhD;1,2 Stephen C. Benson, PhD; ${ }^{3}$ Charles N. Ellis, MD ${ }^{4}$
}

From the Department of Family Medicine, Kern Medical Center, Bakersfield, $C A ;{ }^{1}$ the Department of Family Medicine, University of California, Irvine, Irvine, $C A ;{ }^{2}$ the Department of Biological Sciences, California

State University, Hayward, $\mathrm{CA} ;{ }^{3}$ and the Department of

Dermatology, University of Michigan Medical School, Ann Arbor, $\mathrm{Ml}^{4}$

Address for correspondence: Harrihar A. Pershadsingh, MD,

PhD, Department of Family Medicine, Kern Medical Center, Bakersfield, CA 93305 E-mail: hpershad@uciedu www.lejacq.com ID: 4434

The authors conducted a prospective, open-label, pilot trial of the effects of the antidiabetic thiazolidinedione (TZD) rosiglitazone in two patients with moderate to severe plaque psoriasis. Case 1: A lean, euglycemic 43-year-old nondiabetic man with a 2-year history of plaque psoriasis presented with lesions involving $10 \%$ of his body surface (Figures 1A, 1B, 1C). He had no other chronic or acute medical problems. He had previously been managed sporadically with topical triamcinolone acetonide, an intermediate-strength glucocorticoid, and was off antipsoriatic medication for 5 months. He was started on rosiglitazone p.o., $8 \mathrm{mg}$ q.d. After 10 weeks on rosiglitazone, the lesions developed increased erythema, spreading, and shedding of scale (Figures 2A, 2B, 2C). After an additional 26 weeks, the lesions had largely disappeared (Figures 3A, 3B, 3C). The patient remained euglycemic throughout the study. His liver function enzymes (alanine transferase [ALT] and aspartate transferase $[\mathrm{AST}]$ ) remained normal throughout the study: ALT, $23 \mathrm{IU} / \mathrm{L} ; \mathrm{AST}, 47 \mathrm{IU} / \mathrm{L}$ before treatment; ALT, 25 IU/L; AST, 33 IU/L after treatment. There were no adverse events. Case 2: An overweight 68-year-old woman (body mass index, $29 \mathrm{~kg} / \mathrm{m}^{2}$; with a 12-year history of type 2 diabetes and 5 -year history of psoriasis presented with generalized plaque psoriasis over $20 \%$ of her body, including two large, thick, silvery plaques with the texture of leather over the lower part of the back (Figure 4A). She was given rosiglitazone p.o., $4 \mathrm{mg}$ b.i.d. for 24 weeks, which resulted in significant improvement in psoriasis (Figure 4B). After an additional 26 weeks on rosiglitazone, the plaques had cleared on her back (Figure 4C) and over her entire body, including scalp, ears, and posterior forearms (not shown). Her glycemic control improved (hemoglobin $\mathrm{A}_{1 \mathrm{c}}$ decreased from 7.7\% to 7.2\%) and liver function remained normal throughout the study (ALT, 24 IU/L; AST, $14 \mathrm{IU} / \mathrm{L}$ before treatment; and ALT, $26 \mathrm{IU} / \mathrm{L} ; \mathrm{AST}, 15 \mathrm{IU} / \mathrm{L}$ after treatment). There were no adverse events.

$\mathbf{T}$ he antidiabetic thiazolidinediones (TZDs) represent a new class of insulin-sensitizing antidiabetic agents. ${ }^{1}$ Rosiglitazone and pioglitazone are the only TZDs presently approved for the treatment of type 2 (insulin resistant) diabetes. Rosiglitazone lowers blood glucose by improving insulin sensitivity without increasing circulating insulin concentrations, and therefore is not associated with hypoglycemia when administered to diabetic or nondiabetic patients. Troglitazone, the first in the class to be approved, was withdrawn from the market in 2000 due to idiosyncratic hepatotoxicity. ${ }^{2}$ After 6 years of general use, hepatotoxicity does not appear to be associated with either rosiglitazone or pioglitazone., ${ }^{1,3}$ Fluid retention and peripheral edema have been documented in approximately $5 \%$ of diabetic patients taking TZDs, which increases to an incidence of $15 \%$ in those concurrently using insulin. ${ }^{1,4}$ However, edema does not appear to be a problem in nondiabetics. ${ }^{4}$
Informed consent was obtained from the patients for the new use of these drugs to treat psoriasis, and for publication of the study. The Kern Medical Center Institutional Review Board, which is in compliance with the Helsinki Declaration, approved this study.

The peroxisome proliferator-activated receptor (PPAR) isoforms $\alpha, \gamma$, and $\delta$ belong to the nuclear receptor superfamily that includes receptors for the steroid hormones, thyroid hormone, vitamin $\mathrm{D}$, and retinoids. To be active in gene transcription, PPAR isoforms form a ligand-activated obligate heterodimer with the retinoid X receptor (RXR) (PPARRXR) to regulate access of the core transcriptional machinery to target gene sequences. ${ }^{1}$

The discovery that TZDs were high-affinity activators of PPAR- $\gamma,{ }^{5}$ and that they had potent anti-inflammatory, antiproliferative, and immunomodulatory effects, $s^{4,6}$ led to the speculation that PPAR- $\gamma$ agonists may be 
effective agents in the treatment of chronic inflammatory diseases. TZDs had been shown to down-regulate proinflammatory nuclear transcription factors (e.g., nuclear factor- $\kappa \beta$, nuclear factor of activated T lymphocytes, activator protein-1), proteolytic enzymes (e.g., matrix metalloproteinase-9), inflammatory interleukins (ILs) (e.g., IL-1 $\beta$, IL-2, IL-6) and other inflammatory molecules (e.g., tumor necrosis factor- $\alpha$, inducible NO synthase), and to modulate $\mathrm{T}$ helper $\mathrm{TH} 1 /$ TH2 lymphocyte transition to a more differentiated, less active phenotype. ${ }^{4,6-8}$ PPAR- $\gamma$ agonists exert anti-inflammatory and antiproliferative activities by antagonizing an array of important signaling pathways. ${ }^{4,6-8}$

These proinflammatory and immunostimulatory signaling pathways are known to contribute to the pathophysiology of psoriasis, a proliferative, inflammatory disease of the epidermis in which keratinocyte hyperproliferation and immune dysfunction are major components. ${ }^{9}$ Inappropriate $\mathrm{T}$ lymphocyte activation leads to elaboration of proinflammatory cytokines, tissue inflammation, and keratinocyte hyperproliferation, important pathogenic aspects of psoriasis. The extensive utility of retinoids in the treatment of psoriasis is well documented. Interestingly, retinoid RXR agonists that activate the PPAR/ RXR heterodimer can also increase the insulin sensitivity of diabetic and obese mice, ${ }^{10}$ and other retinoids increase insulin sensitivity in psoriasis patients. ${ }^{11}$ Troglitazone was shown to clinically ameliorate plaque psoriasis in diabetic and nondiabetic individuals. ${ }^{12}$ However, this drug is no longer available. Pioglitazone ameliorated psoriasis in a patient with nonalcoholic steatohepatitis and type 2 diabetes, ${ }^{13}$ but there are no reports of the effectiveness of rosiglitazone, the only other widely prescribed TZD, in psoriatic patients. The two cases presented herein illustrate that oral administration of rosiglitazone at doses approved for the treatment of type 2 diabetes resulted in virtual eradication of their psoriatic lesions without any evidence of adverse effects.

Oral administration of pioglitazone ${ }^{14}$ and rosiglitazone ${ }^{15}$ were shown to reduce carotid intimal-medial thickness in diabetic ${ }^{14}$ and nondiabetic $^{15}$ patients, consistent with retardation of atherosclerotic disease progression; an

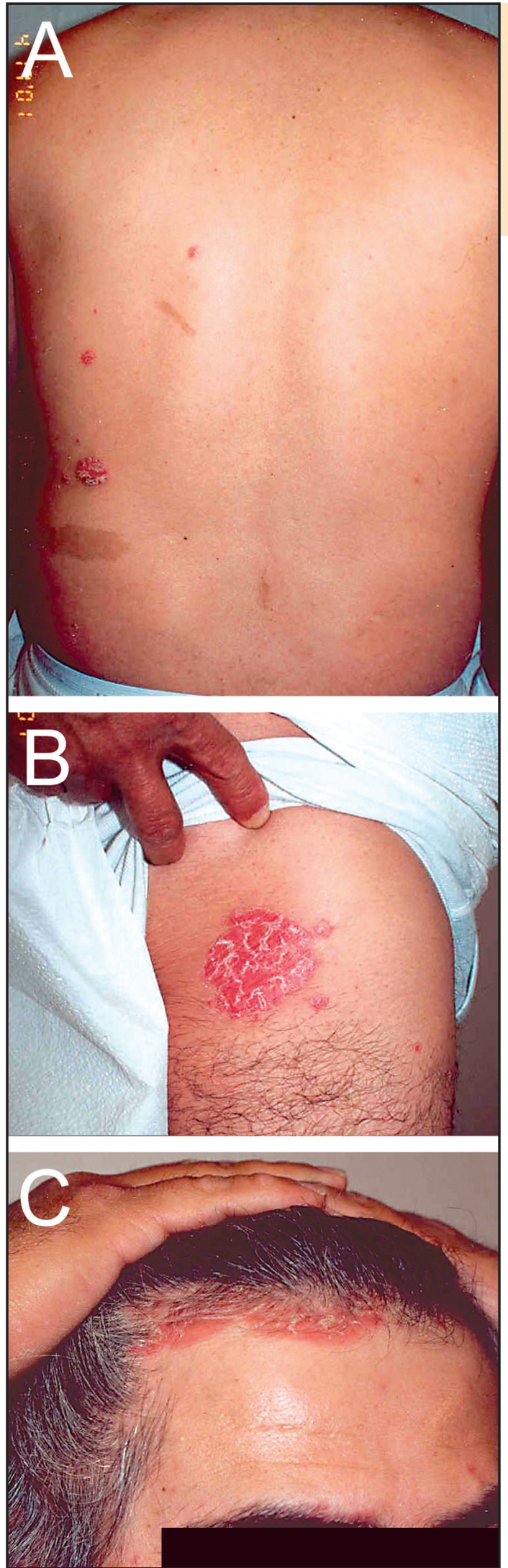

Figure 1. Oral administration of rosiglitazone $(8$ mg daily) improved psoriatic lesions in a nondiabetic patient. Baseline images before therapy: A) left posterior thorax; $B$ ) right outer thigh superiorly; C) forehead 
Figure 2. Psoriatic lesions in Figure 1 A-C after 10 weeks of therapy with rosiglitazone
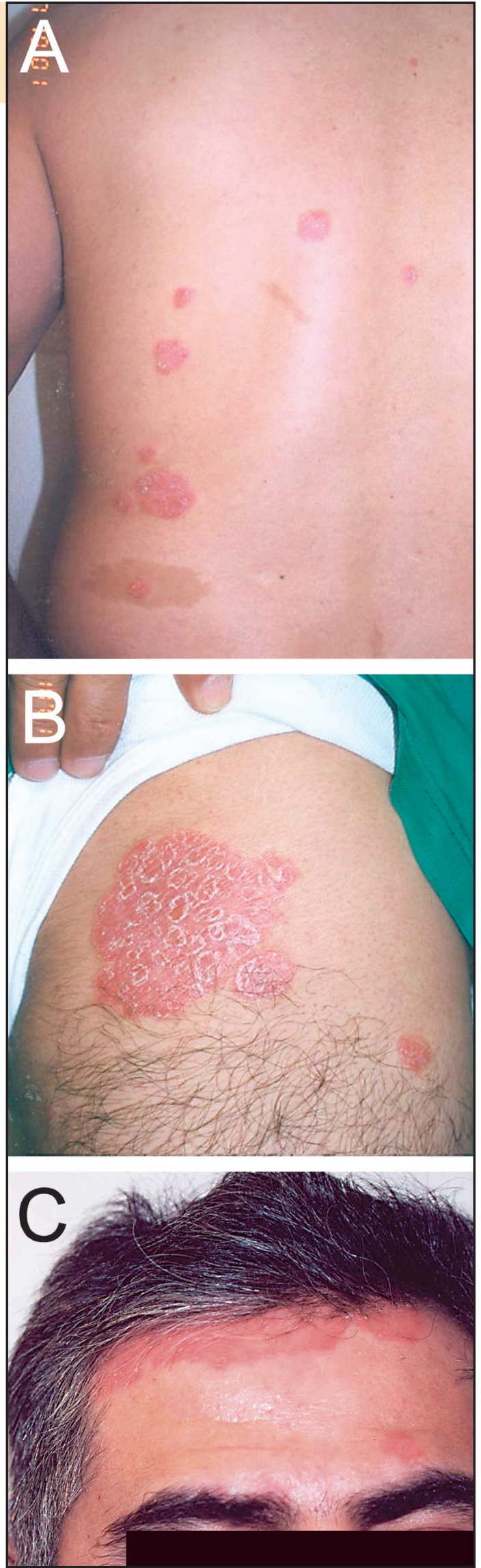

We previously demonstrated the presence of PPAR- $\gamma$ protein and messenger RNA in cultured human keratinocytes, and that troglitazone inhibited the proliferation of keratinocytes derived from nonpsoriatic and psoriatic skin biopsies. ${ }^{12}$ More recently we showed that rosiglitazone also had potent growth-inhibitory effects on keratinocytes in monolayer and in organ cultures derived from human skin. ${ }^{16}$ In contrast to its inhibitory effects on keratinocytes, the proliferation of dermal fibroblasts were unaffected by troglitazone $^{12}$ or rosiglitazone. ${ }^{16}$

In vitro, cultured human peripheral blood mononuclear cells highly enriched in T lymphocytes were induced to secrete IL-2 by exposure to the potent mitogens phytohemagglutinin and phorbol myristate acetate. Incubation with rosiglitazone inhibited phytohemagglutinin-phorbol myristate acetate-induced secretion of IL-2 in a concentration-dependent manner between 0.1 and $10 \mu \mathrm{mol} / \mathrm{L}$ (Table). ${ }^{17}$ Cell viability was unaffected by rosiglitazone. Rosiglitazone had a small but significant inhibitory effect on mitogen-induced IL-2 production in $\mathrm{T}$ lymphocyte-enriched peripheral blood mononuclear cells at $0.1 \mu \mathrm{mol} / \mathrm{L}$; a concentration attainable in blood at doses approved for the treatment of diabetes. ${ }^{18}$

During initial treatment, the clinical responses to rosiglitazone were similar to findings in patients with psoriasis treated with oral retinoids. ${ }^{19}$ Therapy with particular TZDs (e.g., troglitazone vs. rosiglitazone) appear to differ in the time for a clinical response. Troglitazone had an earlier onset of clearing of disease, ${ }^{12}$ with the initial clinical effect being evident within $4-8$ weeks, and almost complete clearing seen after 16-20 weeks. ${ }^{12}$ With rosiglitazone, a beneficial effect was not clearly evident until after 10-26 weeks, with virtually complete clearing seen after 8-12 months in both the nondiabetic (Case 1) and the diabetic (Case 2) patient at the maximum approved dose for treating type 2 diabetes. Whether chronicity of the psoriasis and/or the coexistence of diabetes, a known immunodeficient state, may be related to the extended period of time required for significant clearing of the psoriasis (Case 2) remains to be determined. Additionally, we demonstrate that rosiglitazone suppressed IL-2 release from human peripheral blood 
mononuclear cells enriched in T lymphocytes (Table) at $0.1 \mu \mathrm{mol} / \mathrm{L}$; a concentration that is achievable at the doses utilized in this study. ${ }^{18}$ IL-2 is a key inflammatory cytokine in the pathogenesis of psoriasis and other inflammatory diseases. ${ }^{4}$

These findings suggest that rosiglitazone may be useful for treating psoriasis vulgaris. In general, the TZD class of drugs may be useful for ameliorating inflammatory skin diseases such as psoriasis and atopic dermatitis. 4,12,16 It is important to note that, because they do not function as insulin secretagogues, rosiglitazone and other TZDs are not associated with hypoglycemia. ${ }^{3,4}$ Despite the limitations of these anecdotal findings, the potential use of rosiglitazone and pioglitazone, and possibly other PPAR- $\gamma$ agonists, in the treatment of psoriasis $^{4,20}$ requires randomized clinical trials before these drugs can be recommended in certain subsets or in the majority of psoriatic patients.

Disclosure: Dr. Pershadsingh is a principal and owns stock in Bethesda Pharmaceuticals, Inc., which holds intellectual property related to the use of TZDs for treatment of psoriasis and which has received payments from GlaxoSmithKline, the manufacturer of rosiglitazone. Dr. Ellis has served as a consultant to GlaxoSmithKline.

Acknowledgments: Supported by NIH Grant 2R42AR44767-02A2 (Dr. Pershadsingh), a Joint Venture Grant from California State University Program for Education and Research in Biotechnology (to Dr. Benson), and a grant from Bethesda Pharmaceuticals, Inc.

\section{REFERENCES}

1 Willson TM, Lambert MH, Kliewer SA. Peroxisome proliferator-activated receptor$\gamma$ and metabolic disease. Annu Rev Biochem. 2001;70:341-367.

2 Watkins PB, Whitcomb RW. Hepatic dysfunction associated with troglitazone. N Engl J Med. 1998;338:916-917.

3 Isley WL. Hepatotoxicity of thiazolidinediones. Expert Opin Drug Saf. 2003;2:581-586.

4 Pershadsingh HA. Peroxisome proliferator-activated receptor- $\gamma$ : therapeutic target for diseases beyond diabetes: quo vadis? Expert Opin Investig Drugs. 2004;13:215-228.

5 Lehmann JM, Moore LB, Smith-Oliver TA, et al. An antidiabetic thiazolidinedione is a high-affinity ligand for peroxisome proliferator-activated receptor- $\gamma$ (PPAR- $\gamma)$. J Biol Chem. 1995;270:12953-12956.

6 Ricote M, Li AC, Willson TM, et al. The peroxisome proliferator-activated receptor- $\gamma$ is a negative regulator of macrophage activation. Nature. 1998:391:79-82.

7 Jiang C, Ting AT, Seed B. PPAR- $\gamma$ agonists inhibit production of monocyte inflammatory cytokines. Nature. 1998;391:82-86.

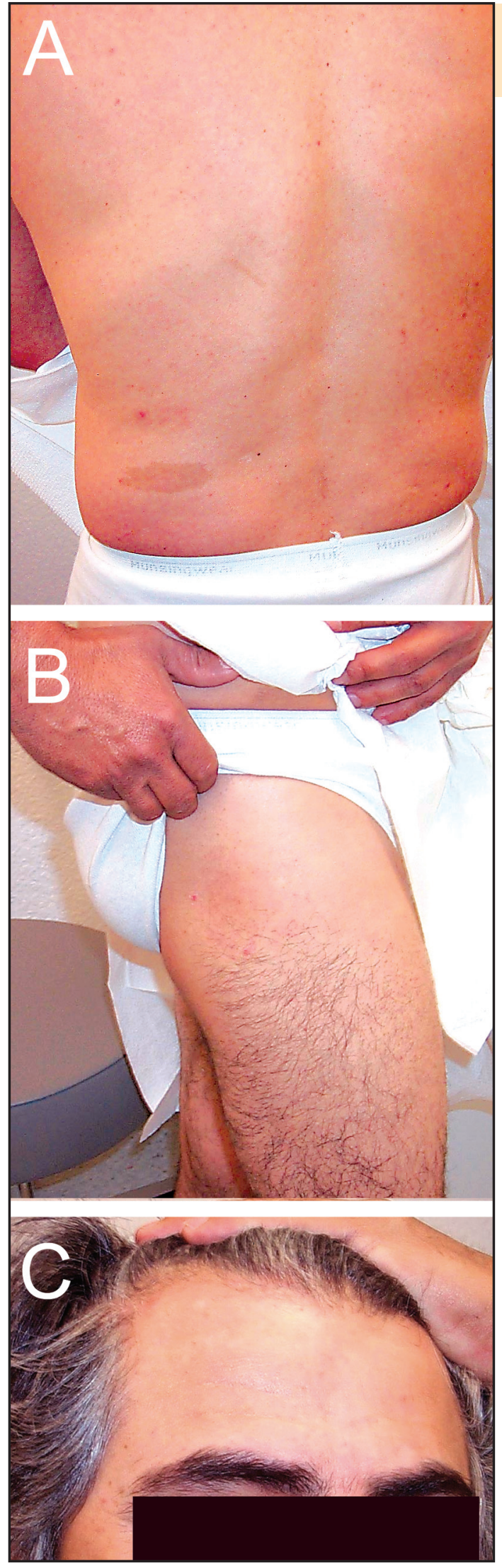

Figure 3. Psoriatic lesions in Figure 1 A-C after an additional 26 weeks' therapy 
Figure 4. Oral administration of rosiglitazone $(4 \mathrm{mg}$ twice daily) improved large psoriatic plaque lesions on the thoracolumbar region of the back in a patient with longstanding type 2 diabetes. A)

Before therapy; B) after 24 weeks of therapy with rosiglitazone; C) after an additional 26 weeks' therapy.
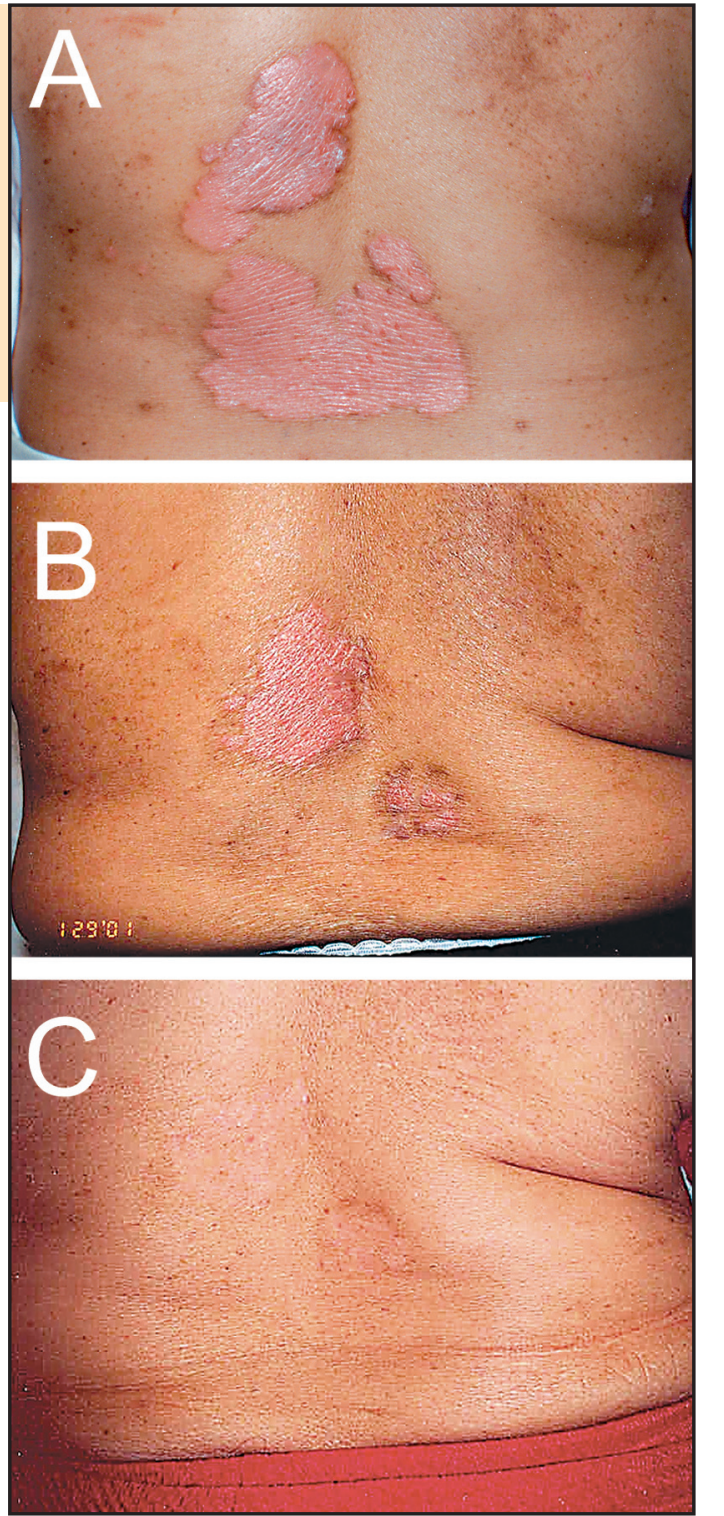

8 Clark RB. The role of PPARs in inflammation and immunity. J Leukoc Biol. 2002;71:388-400. 9 Lebwohl M. Psoriasis. Lancet. 2003;361:1197-1204.

10 Mukherjee $R$, Davies PJ, Crombie DL, et al. Sensitization of diabetic and obese mice to insulin by retinoid $\mathrm{X}$ receptor agonists. Nature. 1997;386:407-410.

11 Ellis CN, Kang S, Vinik AI, et al. Glucose and insulin responses are improved in patients with psoriasis during therapy with etretinate. Arch Dermatol. 1987;123:471-475.

12 Ellis CN, Varani J, Fisher GJ, et al. Troglitazone improves psoriasis and normalizes models of proliferative skin disease: ligands for peroxisome proliferator-activated receptor- $\gamma$ inhibit keratinocyte proliferation. Arch Dermatol. 2000;136:609-616.

13 Itoh S, Kanazuka A, Akimoto T. Combined treatment with ursodeoxycholic acid and pioglitazone in a patient with NASH associated with type 2 diabetes and psoriasis. Dig Dis Sci. 2003;48:2182-2186.

14 Koshiyama H, Shimono D, Kuwamura N, et al. Rapid communication: inhibitory effect of pioglitazone on carotid arterial wall thickness in type 2 diabetes. I Clin Endocrinol Metab. 2001;86:3452-3456.

15 Sidhu JS, Kaposzta Z, Markus HS, et al. Effect of rosiglitazone on common carotid intima-media thickness progression in coronary artery disease patients without diabetes mellitus. Arterioscler Thromb Vasc Biol. 2004;24:930-934.

16 Bhagavathula $N$, Nerusu KC, Lal A, et al. Rosiglitazone inhibits proliferation, motility, and matrix metalloproteinase production in keratinocytes. I Invest Dermatol. 2004;122:130-139.

17 Mosmann T. Rapid colorimetric assay for cellular growth and survival: application to proliferation and cytotoxicity assays. J Immunol Methods. 1983;65:55-63.

18 Hruska MW, Frye RF. Simplified method for determination of rosiglitazone in human plasma. J Chromatogr B Analyt Technol Biomed Life Sci. 2004;803:317-320.

19 Ellis CN, Voorhees JJ. Etretinate therapy. J Am Acad Dermatol. 1987;16:267-291.

20 Kuenzli S, Saurat JH. Peroxisome proliferatoractivated receptors as new molecular targets in psoriasis. Curr Drug Targets Inflamm Allergy. 2004;3:205-211.

Table. Anti-Inflammatory Effects of Rosiglitazone Determined as the Ability to Suppress the Production of Interleukin-2 (IL-2) in MitogenActivated Peripheral Blood Lymphocytes

\begin{tabular}{|lcl|}
\hline EXPERIMENTAL CONDITION & IL-2 RELEASED $(\mathrm{PG} / \mathrm{ML})^{*}$ & PERCENT INHIBITION \\
\hline Control (no drug) & $545 \pm 41$ & \\
Rosiglitazone $(\mu \mathrm{mol} / \mathrm{L})$ & $481 \pm 11^{* *}$ & 12 \\
0.1 & $406 \pm 12^{* *}$ & 25 \\
1.0 & $323 \pm 26^{* *}$ & 41 \\
10 & & \\
\hline
\end{tabular}

T lymphocyte activation was measured as IL-2 production by human peripheral blood mononuclear cells. Cells were isolated from healthy volunteers by Ficoll density gradient centrifugation, cultured in 200:I Roswell Park Memorial Institute 1640 medium supplemented with glutamine $(2 \mathrm{mmol})$, penicillin $\mathrm{G}(100 \mathrm{U} / \mathrm{mL})$, streptomycin $(100 \mathrm{mg} / \mathrm{mL})$ and $5 \%$ fetal bovine serum at $1 \times 10^{5}$ cells per well with or without phytohemagglutinin $(5 \mathrm{mg} / \mathrm{mL})$ and phorbol myristate acetate $(1 \mu \mathrm{mol} / \mathrm{L})$. The media was assayed for IL-2 concentration using a commercial enzyme-linked immunosorbent assay kit (R\&D Systems, Minneapolis, MN). T lymphocyte viability was assessed by 3-(4,5dimethylthiazol-2-yl)-2,5-diphenyl tetrazolium bromide assay. ${ }^{17}$ *Data shown are the mean \pm SEM of four determinations in a representative experiment. ${ }^{* *} p<0.05$ compared with control, using one-way analysis of variance 